\title{
The Effect of a Dispersible Palmitoylethanolamide (Levagen+) Compared to a Placebo for Reducing Joint Pain in an Adult Population - A Randomised, Double-Blind Study
}

\author{
David Briskey ${ }^{1,2}$, Georgia Roche ${ }^{2}$, Amanda $\operatorname{Rao}^{2,3, \text { * }}$ \\ ${ }^{1}$ School of Human Movement and Nutrition Sciences, University of Queensland, Brisbane, Australia \\ ${ }^{2}$ RDC Global, RDC Clinical, Newstead, Brisbane, Australia \\ ${ }^{3}$ School of Medicine, University of Sydney, Sydney, Australia
}

Email address:

d.briskey@uq.edu.au (D. Briskey), georgia@RDCglobal.com.au (G. Roche),Amanda@RDCglobal.com.au (A. Rao)

${ }^{*}$ Corresponding author

\section{To cite this article:}

David Briskey, Georgia Roche, Amanda Rao. The Effect of a Dispersible Palmitoylethanolamide (Levagen+) Compared to a Placebo for Reducing Joint Pain in an Adult Population - A Randomised, Double-Blind Study. International Journal of Nutrition and Food Sciences. Vol. 10, No. 1, 2021, pp. 9-13. doi: 10.11648/j.ijnfs.20211001.12

Received: December 21, 2020; Accepted: December 29, 2020; Published: February 10, 2021

\begin{abstract}
A commonality of all joint pain is the existence of inflammation. Palmitoylethanolamide (PEA) is an endogenous saturated fatty acid derivative that down-regulates multiple proinflammatory and nociceptive pathways and known to inhibit mast and glial cell activity. This study aimed to assess the efficacy of PEA (Levagen+), for alleviating joint pain and improving quality of life in adults. A randomised, double blind, placebo-controlled study on adults reporting joint pain. 74 participants that received either PEA $(n=35)$ or a placebo $(n=39)$ daily for 2 weeks completed this study. The primary outcome was a selfassessed reduction in pain as assessed by a visual analogue scale (VAS) for pain, completed in the morning and evening. VAS pain scores reduced over the 2 weeks of treatment in both groups. Morning VAS scores were significantly reduced from baseline in the PEA and placebo groups from day 3 and 4 respectively. VAS scores were significantly lower in the PEA group compared to the placebo group on day $14(\mathrm{P}<0.05)$. Evening VAS scores were significantly reduced from baseline in both the PEA and placebo groups from day 3 . Total mood scores for both groups were similar at baseline but was significantly different at the end of the study, with the PEA group decreasing and the placebo group increasing. This study demonstrates that PEA may be a safe and effective option for reducing joint pain. Future studies should investigate whether long-term supplementation can show further improvements in pain scores.
\end{abstract}

Keywords: Palmitoylethanolamide, PEA, Levagen, Joint Pain

\section{Introduction}

Joint pain is a common yet complex condition experienced by people of any age to varying degrees [1-4], it is a leading contributor of disability worldwide [5] and significantly contributes to the global burden of disease [6]. Pain can be felt in any joint of the body and can arise for a variety of reasons including; arthritis, infection, autoimmune disease and trauma $[4,5,7,8]$. Joint pain is often accompanied by restricted mobility, therefore limiting daily activities and reducing quality of life [4]. A commonality of all joint pain is the existence of inflammation due to the local release of proinflammatory cytokines $[5,8,9]$. First line therapy to combat joint pain is over-the-counter pain-relieving medications, including paracetamol, non-steroidal anti-inflammatories and opioids [10]. The reliance on such products can result in unwanted gastric, renal and hepatic effects [11, 12], as well as leading to the development of medication tolerance and dependency $[10,13]$. Therefore, the demand for effective and well-tolerated pain relief is increasing.

Palmitoylethanolamide (PEA) is an endogenous saturated fatty acid derivative that exerts anti-inflammatory and 
analgesic properties [14]. In the body, PEA is synthesized from Palmitic acid (C16:0), the most common fatty acid in animals [15]. Synthesis of PEA takes place in membranes of various cell types and produced on demand to act locally [1416]. When cells are subjected to potentially harmful stimuli, they express a selective enzyme that releases PEA from the membrane [15]. Tissue levels of PEA are tightly regulated through a balance between synthesis and breakdown. Since discovered in 1970 as a therapeutic food, the safety of PEA has been tested in a multitude of animal and human populations. An exogenous supramaximal dose of PEA from $300 \mathrm{mg}$ up to $1,200 \mathrm{mg}$ per day has been studied extensively in humans, with no adverse effects reported [17].

The analgesic and anti-inflammatory effects of PEA have been widely researched [16] and supported by several pain studies [12, 17-22]. PEA is reported to down-regulate multiple proinflammatory and nociceptive pathways and is known to inhibit mast and glial cell activity [16, 17, 22]. Recently, a double-blind randomized placebo-controlled study assessed the efficacy of PEA for symptoms of knee osteoarthritis and found that PEA may be a novel treatment for attenuating pain and reducing other associated symptoms of knee osteoarthritis [23]. The aim of this study was to assess the effectiveness of PEA for reducing general joint pain and improving quality of life compared to a placebo.

\section{Main Body}

\subsection{Methods}

\subsubsection{Design}

A randomised, double-blinded placebo-controlled study investigated the efficacy of PEA on joint pain over a 2-week period. The study was conducted in Australia between October 2019 and June 2020 on 80 otherwise healthy adults aged between 25-70 years old experiencing joint pain. This study was conducted in compliance with the current International Conference on Harmonization (ICH) Guideline for Good Clinical Practice (GCP), the Therapeutic Goods Administration (TGA) and was conducted in accordance with ethical approval from Bellberry Limited; an NHMRC accredited Human Research and Ethics Committee and registered on the ANZCTR (ACTRN12619001467123). All participants provided written informed consent and were screened for inclusion and exclusion criteria prior to commencing the study.

\subsubsection{Study Population}

Participants were recruited from mailing list and public media outlets. Potential participants underwent preliminary screening via telephone and were screened for inclusion criteria including: aged between 25-70 years, experiencing joint pain (not associated with acute injury or long-standing disease), generally healthy, able to provide consent, unable to fall pregnant, agreed to not change current diet or exercise regime, and agreed not to take other pain medication during the study. Exclusion criteria included unstable or serious illness, serious mood or neurological disorders, malignancy or treatment for malignancy within the previous two years, diagnosis of rheumatoid arthritis, bursitis or gout, tobacco use, substance abusers, chronic past or current alcohol use, allergy to any ingredients in the active or placebo formula, known pregnant or lactating women, any condition which the investigator believed the participant unsuitable for inclusion, participation in another clinical trial within the previous 1 month, or had a history of infection in the month prior to the study.

\subsubsection{Trial Product}

Participants in the PEA group consumed $175 \mathrm{mg}$ of Levagen $+{ }^{\mathrm{TM}}$ twice daily (morning and night) with water. Levagen $+^{\text {TM }}$ contains PEA with LipiSperse ${ }^{\circledR}$, a cold water dispersion technology and has been shown to increase the absorption of PEA [24]. The placebo group consumed maltodextrin using the same dosing regimen as the PEA group. Both the participants and investigators were blind as to the product allocation, with product allocation conducted using random allocation software. The PEA and placebo product were housed in an opaque bottle, and the placebo appeared identical to the PEA comparator. All participants were provided with enough product to last 14 days.

\subsubsection{Procedure}

Following phone screening, potential participants underwent an information session after which they provided their consent for inclusion in the study. Consenting participants then underwent a health assessment including lifestyle, medication and medical history. Once enrolled, participants were randomly allocated to either the placebo or the active intervention group. Participants had baseline measurements performed before receiving a 2-week supply of the study product. Prior to product allocation, participants completed a baseline pain-recording period. During both the baseline and supplement period, participants recorded pain values using a visual analogue scale (VAS) twice daily. The pain VAS is scale consisting of a 100 $\mathrm{mm}$ line that represents a continuum between "no pain" (score of zero) and "worst pain" (score of 100) [25].

Participants were then asked to consume the allocated product according to the dose prescribed and continued to score their pain levels using the VAS via a secured online webpage provided in the morning and evening for 14 days.

\subsubsection{Outcome Measures}

The primary outcome was the change in joint pain assessed using the VAS. VAS scores were calculated as the change in VAS pain scores from baseline over the two-week period and compared within and between groups. Secondary outcomes included: change in weight and change in quality of life as measured by RAND 36-Item Health Survey (SF36), Profile of Mood States (POMS) questionnaire and gastrointestinal tolerance. All secondary outcomes were assessed at baseline and upon study completion.

\subsubsection{Statistical Analysis}

Statistical analysis was conducted using Graphpad Prism 8.0. Results were tested for normality using a d'agostino and pearson normality test with data then analysed for differences using t-tests. 


\subsection{Results}

Table 1. Participant details.

\begin{tabular}{lll}
\hline & PEA & Placebo \\
\hline Participants $(\mathrm{n})$ & 35 & 39 \\
Females $(\mathrm{n})$ & 21 & 26 \\
Males $(\mathrm{n})$ & 14 & 13 \\
Age $($ years; mean $\pm \mathrm{SD})$ & $53.1(10.3)$ & $55.5(10.3)$ \\
Weight $(\mathrm{kg} ;$ mean $\pm \mathrm{SD})$ & $84.5(16.7)$ & $85.7(25.5)$ \\
BMI $\left(\mathrm{kg} / \mathrm{m}^{2}\right)$ & 27.7 & 27.4 \\
\hline
\end{tabular}

$\mathrm{PEA}=$ Palmitoylethanolamide; $\mathrm{BMI}=$ Body Mass Index.

Of the 80 participants enrolled in the study, 74 participants completed the study and were included in the analysis. Six

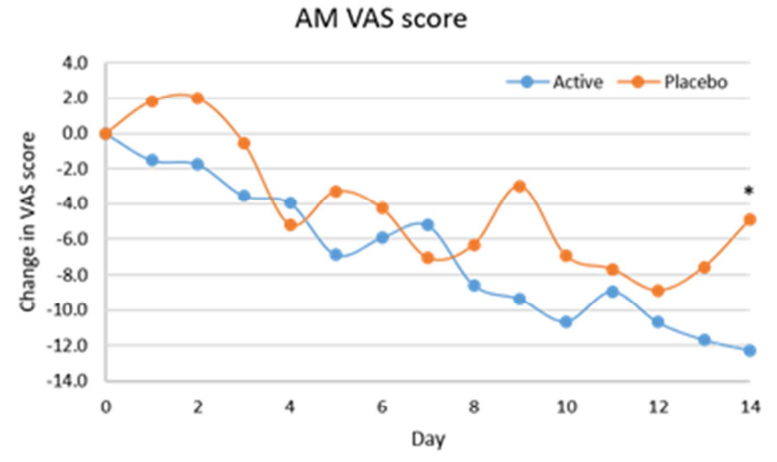

A participants withdrew from the study after completing only the baseline data collection. There were no significant differences between groups at baseline (Table 1) and both groups were normally distributed.

Baseline pain scores were similar in both groups and reduced over the 2 weeks of treatment (table 2). Morning VAS score significantly reduced from baseline in the PEA group $(\mathrm{p}<0.05)$ from day 3 , and the placebo group from day $4(p<0.05)$. Evening VAS scores were significantly lower from baseline in both groups compared to baseline from day 3. The change in morning and evening VAS scored were significantly different between groups at day $14(\mathrm{p}=0.037$ and $\mathrm{p}=0.044$ respectively; Table 2, Figure 1).

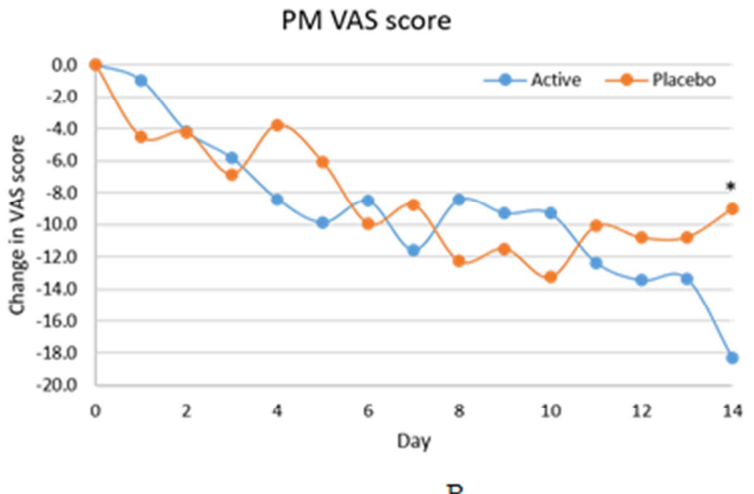

B

Figure 1. Change in VAS score from baseline for morning (A) and evening (B) over 2 weeks. * significant difference between groups $p<0.05$.

Table 2. Pain scores as measure by Visual Analogue Scale.

\begin{tabular}{lllll}
\hline & Morning VAS & \multicolumn{3}{l}{ Evening VAS } \\
\cline { 2 - 5 } & PEA & Placebo & PEA & Placebo \\
\hline Baseline & $42.9(21.7)$ & $38.4(18.9)$ & $46.9(21.5)$ & $43.45(18.9)$ \\
Day 1 & $41.8(23.7)$ & $42.0(21.0)$ & $46.5(25.2)$ & $40.9(17.9)$ \\
Day 2 & $41.1(21.1)$ & $40.3(22.7)$ & $42.3(24.6)$ & $40.5(21.3)$ \\
Day 3 & $39.1(21.4)$ & $39.5(19.3)$ & $39.4(25.2)$ & $37.1(19.2)$ \\
Day 4 & $37.7(24.5)$ & $32.9(19.3)$ & $38.8(23.8)$ & $40.2(21.8)$ \\
Day 5 & $34.8(21.8)$ & $36.1(22.1)$ & $36.8(22.7)$ & $39.4(21.6)$ \\
Day 6 & $35.8(20.2)$ & $34.6(22.3)$ & $38.5(20.5)$ & $35.3(23.1)$ \\
Day 7 & $37.6(20.9)$ & $31.4(21.3)$ & $36.4(20.0)$ & $31.1(17.6)$ \\
Day 8 & $33.0(20.3)$ & $32.7(21.7)$ & $39.1(21.1)$ & $32.4(20.4)$ \\
Day 9 & $32.8(19.4)$ & $37.1(22.5)$ & $35.2(21.4)$ & $30.6(21.0)$ \\
Day 10 & $34.1(18.9)$ & $31.1(22.5)$ & $34.8(20.7)$ & $29.0(18.5)$ \\
Day 11 & $32.7(18.1)$ & $31.6(24.2)$ & $32.4(17.7)$ & $32.6(20.6)$ \\
Day 12 & $32.3(18.1)$ & $28.5(21.7)$ & $30.6(18.5)$ & $32.3(21.1)$ \\
Day 13 & $30.1(19.5)$ & $30.6(22.6)$ & $30.4(18.9)$ & $31.9(21.0)$ \\
Day 14 & $29.1(20.1)^{*}$ & $33.0(22.3)$ & $28.7(17.5)^{*}$ & $34.1(23.1)$ \\
\hline
\end{tabular}

Baseline values are an average of 3 consecutive days of scoring; VAS = Visual Analogue Scale; PEA = palmatoyelethanolamide; Values presented are mean $(\mathrm{SD})$; * significant difference between groups $\mathrm{p}<0.05$

There were no differences in the RAND 36-item Health Survey (SF-36) at baseline or day 14. For the POMS, total mood score (TMS) was similar at baseline (94.0 PEA and 90.4 Placebo). The change in TMS score at the end of the study compared to baseline was significantly different (higher) in the placebo group only. There was a significant difference between groups at the end of the study for TMS score, with the PEA group decreasing to 92.5 and the placebo group increasing to 96.8 . Both the PEA and placebo products were well tolerated with no adverse gastrointestinal events reported.

\subsection{Discussion}

This randomised, double-blind study compared the efficacy of a $350 \mathrm{mg}$ dose of PEA (175 mg morning and night) to a placebo on relieving joint pain in healthy adults aged between 25 and 70 years. The results show PEA to be effective in reducing joint pain over a 2 -week period. These results are supported by previous studies showing PEA's ability to reduce joint pain symptoms and chronic pain [12, 20, 23].

As previously shown [26], a strong placebo effect was observed in this study. The placebo effect was evident from the beginning of the trial, with both groups demonstrating an early reduction in pain scores compared to baseline values (Figure 1). The reduction in pain scores continued in both groups until approximately day 10 . From day 10 , the pain scores plateaued and increased in the placebo group while continuing to decrease in the PEA group for both morning and evening VAS scores (Figure 1). The pattern for VAS score reduction observed in this is similar to that previously shown in another PEA study [12].

The study by Marini and colleagues [12] indicated a 600$900 \mathrm{mg}$ per day dose of PEA was superior to ibuprofen for reducing temporomandibular joint inflammatory pain in a similar pattern to that observed in this study. However, Marini and colleagues showed a greater reduction in VAS 
scores over the 2-week supplementation period. This may be due to a number of factors. Firstly, Marini supplemented with 2.5 and 1.7 times more PEA in the first and second week respectively compared to the present study. Secondly, the specificity of the joint pain assessed may make the alleviation of perceived pain easier to modify. Our study included a group of individuals experiencing general joint pain and this may be harder to modify due to a possibility of varying aetiologies. A third possible reason for the difference in VAS scores between studies is the VAS starting value. The study conducted by Marini and colleagues started off at a score of 70, almost double the study presented here. Taken together though, these studies demonstrate the ability of PEA to reduce joint pain.

In addition to measuring the perceived joint pain, quality of life was also measured, as this is often a more meaningful measure. This study however found no differences in the overall quality of life questionnaire (SF-36) between treatment groups, although the population were generally healthy and scored in the higher range. The POMS TMD score however, decreased from baseline in the PEA group and increased in the placebo group, indicating an improvement in mood in the PEA group. Possibly the greatest limitation affecting a greater change in the quality of life is the study duration. A longer study duration may be necessary to affect the quality of life to an extent that it becomes significant.

As previously mentioned, PEA exerts its analgesic and anti-inflammatory effects by down-regulating multiple proinflammatory and nociceptive pathways and is known to inhibit mast and glial cell activity $[14,16,17,22]$. The commonly prescribed pharmaceuticals for pain relief generally provide symptomatic relief, without resolving the underlying mechanism of pain [11]. Thus, resulting in continuous use with potentially harmful effects. Opioid use can cause constipation, respiratory depression, impaired cognitive ability and immune suppression, as well as tolerance and dependence when used to treat chronic pain [27]. NSAIDS can also have unwanted gastric, renal and hepatic side effects with chronic use [11]. Joint pain is often a chronic condition and therefore there is a need for an effective and safe treatment that can be used long-term.

The safety profile of PEA has been well researched for over 50 years and evidence suggests that doses up to 1,200 $\mathrm{mg}$ /day have no harmful effects [16]. Our study supports these findings demonstrating a good tolerability to PEA with no adverse effects reported from daily supplementation of $350 \mathrm{mg} /$ day for 2 weeks. However, although our study used a lower dose of PEA the findings are consistent to that of other studies that dosed between $600-800 \mathrm{mg} /$ day $[12,18,19]$.

The primary reason for the lower dose of PEA used in this study compared to previous literature is the addition of the dispersion technology, LipiSperse (sold as Levagen+). Previous research on Levagen+ found that it increases plasma PEA concentrations by 1.75 times that of the standard PEA formulation, allowing PEA to be supplemented at a lower dose for the same absorption [24]. Therefore, the dose used in this study may be able to achieve a similar absorption to that of a $600 \mathrm{mg}$ dose of standard PEA.

A major limitation to this study was that it was disrupted by the COVID-19 pandemic. The original study design had to change for safety reasons, so the study could be completed remotely with no in-clinic visits. Instead, the investigators guided the participants through the study via telephone consultations, and online questionnaires. The original study design planned to investigate changes in systemic inflammation (blood cytokines), which may have supported the observed change in VAS scores. A second limitation to this study is the study duration. Although statistical significance was achieved between groups at the end of the study, it can be seen that the placebo effect only started to dissipate towards the end of the supplementation period. Had the study continued for an additional 7-14 days, it may have shown a greater difference between the two groups. A further limitation to the study may be the lack of information collected on the type of joint pain experienced by each participant. However, as the inclusion criteria ruled out certain conditions and the aetiology of generic joint pain is primarily an underlying inflammatory response, it was considered that this was not required.

\section{Conclusion}

In conclusion, this randomised, double-blind placebocontrolled study demonstrated the efficacy of PEA at reducing both morning and evening joint pain scores compared to a placebo over 14 days. It also showed the safety and efficacy of daily PEA supplementation in healthy adults. Suggesting that PEA may be a safe and beneficial treatment in joint pain management. Limitations to the study included a short study duration, and interruptions due to the COVID-19 pandemic. Further research would benefit from a duration of at least 4 weeks.

\section{References}

[1] Grime, J., J. C. Richardson, and B. N. Ong, Perceptions of joint pain and feeling well in older people who reported being healthy: a qualitative study. Br J Gen Pract, 2010. 60: p. 597603.

[2] Amoako, A. O. and G. G. Pujalte, Osteoarthritis in young, active, and athletic individuals.. Clin Med Insights Arthritis Musculoskelet Disord, 2014. 7: p. 27-32.

[3] Loeser, R. F., Age-related changes in the musculoskeletal system and the development of osteoarthritis. Clin Geriatr Med, 2010. 26: p. 371-386.

[4] Neogi, T., The epidemiology and impact of pain in osteoarthritis. Osteoarthritis and cartilage, 2013. 21(9): p. 1145-1153.

[5] Havelin, J. and T. King, Mechanisms underlying bone and joint pain. Current Osteoporosis Reports, 2018. 16(6): p. 763771. 
[6] Blyth, F. M., et al., The Global Burden of Musculoskeletal Pain-Where to From Here? American journal of public health, 2019. 109(1): p. 35-40.

[7] Chang, A. Y., et al., Frequency of Chronic Joint Pain Following Chikungunya Virus Infection. Arthritis Rheumatol, 2018. 70(4): p. 578-584

[8] Punzi, L., et al., Post-traumatic arthritis: overview on pathogenic mechanisms and role of inflammation. RMD Open, 2016. 2(2): p. 1-9.

[9] Schaible, H. G., et al., Joint Pain. Experimental brain research, 2009. 196(1): p. 153-162.

[10] van Laar, M., et al., Pain treatment in arthritis-related pain: beyond NSAIDs.. The open rheumatology journal, 2012. 6: p. 320-330.

[11] Cazacu, I., C. Mogosan, and F. Loghin, Safety issues of current analgesics: an update.. Clujul medical, 2015. 88(2): p. 128-136.

[12] Marini, I., et al., Palmitoylethanolamide versus a nonsteroidal anti-inflammatory drug in the treatment of temporomandibular joint inflammatory pain. Journal of orofacial pain, 2012. 26(2): p. 99-104.

[13] Tehrani, M., M. Aguiar, and J. D. Katz, Narcotics in rheumatology. Health services insights, 2013. 6: p. 39-45.

[14] Alhouayek, M. and G. G. Muccioli, Harnessing the antiinflammatory potential of palmitoylethanolamide. Drug discovery today, 2014. 19(10): p. 1632-1639.

[15] Keppel Hesselink, J. M., T. de Boer, and R. F. Witkamp, Palmitoylethanolamide: $A$ natural body-own antiinflammatory agent, effective and safe against influenza and common cold.. International journal of inflammation, 2013. 2013.

[16] Keppel Hesselink, J. M., Professor Rita Levi-Montalcini on nerve growth factor, mast cells and palmitoylethanolamine, and endogenous anti-inflammatory and analgesic compound. Journal of Pain Relief, 2013. 2(1).

[17] Gabrielsson, L., S. Mattsson, and C. J. Fowler, Palmitoylethanolamide for the treatment of pain: pharmacokinetics, safety and efficacy. British journal of clinical pharmacology, 2016. 82(4): p. 932-942.

[18] Canteri, L., S. Petrosino, and G. Guida, Reduced consumption of antiinflammatory and analgesic agents in the treatment of chronic neuropathic pain in patients affected by compressioncausing lumbosciatic pain and treated with Normast ${ }^{\circledR} 300$ $m g$. DOLOR, 2010. 25(4): p. 227-234.

[19] Domínguez, C., et al., N-palmitoylethanolamide in the treatment of neuropathic pain associated with lumbosciatica. Pain Management, 2012. 2(2): p. 119-124.

[20] Germini, F., et al., N-of-1 Randomized Trials of UltraMicronized Palmitoylethanolamide in Older Patients with Chronic Pain. Drugs \& Aging, 2017. 34: p. 941-952.

[21] Keppel Hesselink, J. M. and T. Hekker, Therapeutic utility of palmitoylethanolamide in the treatment of neuropathic pain associated with various pathological conditions: a case series. Journal of Pain Research, 2012. 5: p. 437-442.

[22] Skaper, S. D., et al., Palmitoylethanolamide, a naturally occurring disease-modifying agent in neuropathic pain. Inflammopharmacology, 2014. 22(2): p. 79-94.

[23] Steels, E., et al., A double-blind randomized placebo controlled study assessing safety, tolerability and efficacy of palmitoylethanolamide for symptoms of knee osteoarthritis. Inflammopharmacology, 2019. 27(3): p. 475-485.

[24] Briskey, D., A. R. Mallard, and A. Rao, Increased absorption of palmitoylethanolamide using a novel dispersion technology system (LipiSperse $\left.{ }^{\circledR}\right)$. Journal nutraceuticals and food science, 2020. 5(2:3): p. 1-6.

[25] Delgado, D. A., et al., Validation of Digital Visual Analog Scale Pain Scoring With a Traditional Paper-based Visual Analog Scale in Adults. Journal of the American Academy of Orthopaedic Surgeons., 2018. 2(3).

[26] Vase, L. and K. Wartolowska, Pain, placebo, and test of treatment efficacy: a narrative review. British journal of anaesthesia, 2019. 123(2): p. e254-e262.

[27] Benyamin, R., et al., Opioid complications and side effects. Pain physician, 2008. 11: p. S105-S120. 